\title{
氧化银纳米颗粒修饰碳纤维电极用于水果中pH 在线检测
}

\author{
李心如, 周璘, 苏彬*
}

浙江大学化学系, 杭州 310058

*通讯作者, E-mail: subin@zju.edu.cn

收稿日期: 2020-09-30; 接受日期: 2020-11-05; 网络版发表日期: 2020-11-12

国家重点基础研究发展规划(编号: 2019YFC1604504)和国家自然科学基金(编号: 21874117)资助项目

摘要 $\mathrm{pH}$ 检测在农业生产、食品加工、环境保护、疾病诊断等领域有着重要意义。电化学法具有响应速度 快、灵敏度高和操作简单等优点，是目前最常用的 $\mathrm{pH}$ 检测方法之一。然而，商品化的 $\mathrm{pH}$ 计存在体积大、质子敏 感膜易损等问题, 仅能在相对稳定的样本溶液环境中工作, 并不适用于植入式pH分析. 本文将氧化铱纳米颗粒修 饰在碳纤维微电极表面, 构建了一种微型、可植入的固态 $\mathrm{pH}$ 传感器. 该传感器具有超能斯特 $\mathrm{pH}$ 响应, 可以达到约 $65.5 \mathrm{mV} / \mathrm{pH}$ 的灵敏度, 宽检测范围 $(\mathrm{pH} \approx 2-12)$ 和较高稳定性. 最后我们将该 $\mathrm{pH}$ 传感器植入水果组织内, 实现了水 果内 $\mathrm{pH}$ 值的在线检测.

关键词氧化铱, 植入式, $\mathrm{pH}$, 超能斯特, 水果

\section{1 引言}

国际纯粹与应用化学联合会(International Union of Pure and Applied Chemistry, IUPAC)对pH的定义为 溶液中氢离子活度的负指数 ${ }^{[1]} . \mathrm{pH}$ 检测在农业生产、 食品加工、环境保护、疾病诊断等领域有重要意 $义^{[2-8]}$. 以果蔬培育为例, 通过检测果蔬 $\mathrm{pH}$, 农业生产 人员可以快速判断果蔬的成熟度 ${ }^{[2]}$; 在食品加工领域, $\mathrm{pH}$ 能够反映食物中微生物的生长情况，评估食品保鲜 度 ${ }^{[3]}$; 此外, 通过检测水体 $\mathrm{pH}$ 可以快速评估水体污染程 度 $^{[5,6]}$.

电化学方法是一种根据溶液的电参量(如电位、 电阻等)和溶液化学性质的关系建立的分析方法. 电化
学法具有响应速度快、检测范围宽、灵敏度高等优 点, 是目前最常用的 $\mathrm{pH}$ 检测方法 ${ }^{[9]}$. 其中, 玻璃电极被 广泛应用于水溶液的 $\mathrm{pH}$ 检测, 但是, 玻璃电极存在体 积较大、质子敏感玻璃膜易损坏等缺点, 不适用于食 品、植物组织内的 $\mathrm{pH}$ 检测 ${ }^{[10,11]}$. 近年来，离子敏感的 金属/金属氧化物, 例如二氧化钉 $\left(\mathrm{RuO}_{2}\right)^{[12]}$ 、五氧化二 钽 $\left(\mathrm{Ta}_{2} \mathrm{O}_{5}\right)^{[13]}$ 、氧化锡 $\left(\mathrm{SnO}_{2}\right)^{[14]}$ 、二氧化钛 $\left(\mathrm{TiO}_{2}\right)^{[15]}$ 、 氧化铱 $\left(\mathrm{IrO}_{x}\right)^{[16,17]}$ 等在 $\mathrm{pH}$ 检测领域得到了广泛的关注. 金属/金属氧化物可以被修饰在不同类型的基底电极 表面(包括柔性电极和微电极)制成不同类型的固态 $\mathrm{pH}$ 传感器. 固态 $\mathrm{pH}$ 传感器无需内充电解液、体积灵活可 控、可微型化和柔性化 ${ }^{[8,16]}$, 在食品安全、疾病诊断 及环境保护等领域受到广泛关注 ${ }^{[9]}$. 例如, $\mathrm{CaO}$ 等 ${ }^{[18]}$ 将

引用格式: Li X, Zhou L, Su B. Iridium oxide nanoparticles modified carbon fiber microelectrodes for pH detection. Sci Sin Chim, 2021, 51: 383-387, doi: 10.1360/ SSC-2020-0197 
氧化铱修饰在柔性基底表面，结合无线数据传输技术， 构建的可植入式pH传感器用于胃食管反流患者病情 监测. 其中, 氧化铱具有导电率高、稳定性好和生物兼 容性好等优点, 是目前较为理想的 $\mathrm{pH}$ 传感器功能材 料 ${ }^{[19,20]}$. 除此之外, 氧化铱在溶液环境中主要以水化 氢氧化物的形式存在, 在 $\mathrm{pH}$ 检测中常展现出更高的灵 敏度 ${ }^{[21]}$.

在本文中, 我们以碳纤维微电极(carbon fiber microelectrode, CFME) 为基底电极, 通过电化学沉积法在 碳纤维表面修饰氧化铱纳米颗粒(Iridium oxide nanoparticles, IONPs), 制备了一种微型、植入式的pH传感 器. 该传感器的 $\mathrm{pH}$ 响应灵敏度可以达到约 $65.5 \mathrm{mV} / \mathrm{pH}$, $\mathrm{pH}$ 检测范围约为2 12. 最后我们使用该电极实现了西 瓜、橘子组织内 $\mathrm{pH}$ 的在线检测.

\section{2 实验部分}

\section{1 试剂与仪器}

试剂: 四氯化铱水合物 $\left(\mathrm{IrCl}_{4} \cdot \mathrm{H}_{2} \mathrm{O}, \mathrm{Ir}>63.9 \%\right)$, 过氧 化氢 $\left(\mathrm{H}_{2} \mathrm{O}_{2}, 30\right.$ wt.\%)购自美国Sigma公司, 氯化钾 $(\mathrm{KCl}$, $99.5 \%)$, 盐酸 $(\mathrm{HCl}, 36$ wt.\%), 氢氧化钠 $(\mathrm{NaOH}, 96 \%)$, 草酸 $\left((\mathrm{COOH})_{2} \cdot 2 \mathrm{H}_{2} \mathrm{O}, 98 \%\right)$, 无水碳酸钾 $\left(\mathrm{K}_{2} \mathrm{CO}_{3}\right.$, 99.5\%)购自国药集团试剂有限公司，碳纤维购自日本 Toray公司, 导电银胶 $(6 \mathrm{~g})$ 购自美国Humi Seal公司. 所 有溶液均用超纯水 $(18.2 \mathrm{M} \Omega \mathrm{cm}$ )配制. 仪器: 超纯水器 (Milli-Q，Milipore，美国)，玻璃拉制机(PC-100，Narishige, 日本), $\mathrm{pH}$ 计 (Five Easy, Mettler-Toledo, 瑞士), 电 化学工作站(CHI 920, 辰华, 上海), 扫描电子显微镜 (SU-8020, Hitachi, 日本).

\section{2 实验方法}

前驱体溶液的配制：本文中氧化铱前驱体溶液的 配制方法参考Yamanaka ${ }^{[22]}$ 关于电化学修饰氧化铱的 报道. 首先, 在 $50 \mathrm{~mL}$ 超纯水中依次加入 $0.0812 \mathrm{~g} \mathrm{IrCl}_{4}$. $\mathrm{H}_{2} \mathrm{O}, 0.5 \mathrm{~mL} \mathrm{H} \mathrm{H}_{2} \mathrm{O}_{2}$ 和 $0.5 \mathrm{~g}(\mathrm{COOH})_{2} \cdot 2 \mathrm{H}_{2} \mathrm{O}$, 搅拌约 $10 \mathrm{~min}$. 然后，向混合溶液中加入 $\mathrm{K}_{2} \mathrm{CO}_{3}$ 粉末，调节溶 液 $\mathrm{pH}$ 至10.5. 此时溶液呈亮黄色. 将混合溶液置于 $60^{\circ} \mathrm{C}$ 水浴中避光加热 $3 \mathrm{~h}$, 最终可以得到深蓝色前驱体溶液.

氧化铱纳米颗粒修饰碳纤维电极的制备: 首先, 向 前驱体溶液中通入氮气约10 15 min，除去溶液中的氧 气. 并用冰水浴控制溶液温度约为 $10^{\circ} \mathrm{C}$. 然后, 将碳纤
维微电极 (长度约 $1 \mathrm{~mm}$ )作为工作电极, 饱和银/氯化银 电极作为参比电极, 铂丝作为对电极, 将电极浸于氧化 铱前驱体溶液中, 采用计时电流法将氧化铱纳米颗粒 沉积在碳纤维微电极表面. 电流密度为 $0.016 \mathrm{~A} / \mathrm{cm}^{2}$, 沉积时间为 $400 \mathrm{~s}$.

$\mathrm{pH}$ 检测: 我们采用两电极体系, 通过测量开路电 位(open circuit potential, OCP)的方法检测溶液和水果 组织中的 $\mathrm{pH}$. (1) 溶液环境中的 $\mathrm{pH}$ 检测: 将氧化铱纳米 颗粒修饰碳纤维电极作为工作电极, 饱和银/氯化银电 极作为参比电极. 首先, 将氧化铱纳米颗粒修饰碳纤维 电极和饱和银/氯化银电极浸入 $100 \mathrm{~mL} 0.1 \mathrm{~mol} / \mathrm{L} \mathrm{KCl}$ 溶液中。然后，通过滴加不同浓度的 $\mathrm{HCl}$ 溶液和 $\mathrm{NaOH}$ 溶液调节溶液 $\mathrm{pH}$, 同时用电化学工作站记录开路电位. (2) 水果组织中的 $\mathrm{pH}$ 检测: 将氧化铱纳米颗粒修饰碳 纤维电极作为工作电极, 饱和银/氯化银微电极作为参 比电极. 首先用立体定位仪将氧化铱纳米颗粒修饰碳 纤维电极和饱和银/氯化银微电极同时植入水果组织 中, 电极间距约 $5 \mathrm{~mm}$, 稳定 5 10 min后, 用电化学工作 站记录开路电位.

$\mathrm{pH}$ 循环检测实验: 我们采用两电极体系, 将修饰氧 化铱纳米颗粒的碳纤维电极依次浸泡在 $\mathrm{pH}=3$ 和 $\mathrm{pH}=10$ 的 $0.1 \mathrm{~mol} / \mathrm{L} \mathrm{KCl}$ 溶液中, 循环测量开路电位, 观察开路 电位随循环次数的变化情况, 以此评估电极的稳定性.

\section{3 结果与讨论}

\section{1 氧化铱纳米颗粒修饰碳纤维电极的制备和表 征}

我们采用计时电流法在碳纤维微电极表面修饰氧 化铱纳米颗粒, 并对沉积时间进行了优化, 实验结果如 图S1所示. 当沉积时间小于 $200 \mathrm{~s}$ 时，电极对 $\mathrm{pH}$ 基本无 响应, 沉积时间为 $200 \mathrm{~s}$ 和 $400 \mathrm{~s}$ 的电极对 $\mathrm{pH}$ 的响应灵敏 度分别为 $49 \mathrm{mV} / \mathrm{pH}$ 和 $65 \mathrm{mV} / \mathrm{pH}$. 因此, 我们选择 $400 \mathrm{~s}$ 为后续实验的沉积时间.

我们对氧化铱纳米颗粒修饰前后的电极进行了扫 描电子显微镜表征. 如图 $1(a, b)$ 所示, 氧化铱纳米颗粒 均匀的分布在碳纤维电极表面, 粒径约为420 520 nm.

为进一步评估电化学沉积的氧化铱纳米颗粒的存 在状态，我们对氧化铱纳米颗粒进行了X射线衍射表 征. 结果如图S2所示, 与氧化铱 $\left(\mathrm{IrO}_{2}\right)$ 的标准谱相比, 电化学方法制备的氧化铱的衍射峰强度较小且难以分 

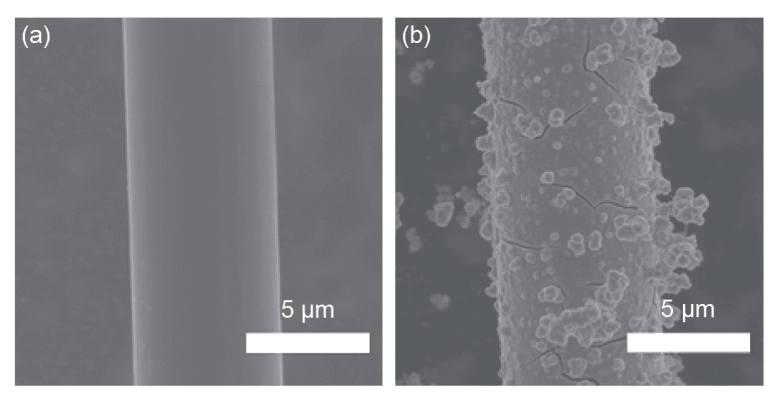

图 1 碳纤维微电极(a)和氧化铱纳米颗粒修饰碳纤维电极 (b)的扫描电子显微镜图 (网络版彩图)

Figure 1 Scanning electron microscope images of carbon fiber microelectrode (a) and iridium oxide nanoparticles modified carbon fiber microelectrode (b) (color online).

峰, 说明通过电化学方法修饰在碳纤维电极表面的氧 化铱纳米颗粒呈无定形状态 ${ }^{[16,23]}$.

\section{2 氧化铱纳米颗粒修饰碳纤维电极用于pH检测}

我们采用两电极体系, 将氧化铱纳米颗粒修饰碳 纤维电极作为工作电极, 饱和银/氯化银电极作为参比 电极，通过测量开路电位的方法对氧化铱纳米颗粒修 饰碳纤维电极的 $\mathrm{pH}$ 检测性能进行表征. 结果如图2所 示, 氧化铱纳米颗粒修饰碳纤维电极对溶液 $\mathrm{pH}$ 变化具 有超能斯特响应, 灵敏度为 $65.5 \mathrm{mV} / \mathrm{pH}$, 线性范围约为 2 12. 为评估氧化铱修饰碳纤维电极检测 $\mathrm{pH}$ 的重复性, 我们用 10 根氧化铱修饰的碳纤维电极分别检测 $\mathrm{pH}=3$ 和 $\mathrm{pH}=10$ 的 $0.1 \mathrm{M} \mathrm{KCl}$ 溶液的开路电位. 结果如图S3a所 示, 电极在 $\mathrm{pH}=3$ 和 $\mathrm{pH}=10$ 的 $0.1 \mathrm{~mol} / \mathrm{L} \mathrm{KCl}$ 溶液中测得 的开路电位分别为 $0.37 \pm 0.02 \mathrm{~V}$ 和 $-0.04 \pm 0.01 \mathrm{~V}(n=10)$, 说明该电极检测 $\mathrm{pH}$ 具有较好的重复性.

为了解释氧化铱纳米颗粒修饰碳纤维电极的超能 斯特 $\mathrm{pH}$ 响应, 我们分析了氧化铱纳米颗粒修饰碳纤维 电极的 $\mathrm{pH}$ 响应机理. 金属氧化物对 $\mathrm{pH}$ 的响应一般来源 于和金属氧化物结合的水分子对金属氧化物的氢化作 用. 通过溅射法、热氧化法等方法修饰在电极表面的 氧化铱结构致密且无水. 因此, 制备的 $\mathrm{pH}$ 传感器的灵 敏度符合经典能斯特方程 $(59 \mathrm{mV} / \mathrm{pH})^{[21]}$. 而采用电化 学方法制备的氧化铱大多以水化氢氧化物的形式存 在, 将这种水化氢氧化物浸入溶液中时, 水化氢氧化物 表面可以形成水化层, 促进其与氢离子的相互作用, 使 $\mathrm{pH}$ 传感器的灵敏度超过 $59 \mathrm{mV} / \mathrm{pH}^{[24]}$. 本文中, 借助电 化学法制备的氧化铱纳米颗粒修饰碳纤维电极的灵敏 度为 $65.5 \mathrm{mV} / \mathrm{pH}$, 我们推测对应的反应过程为:
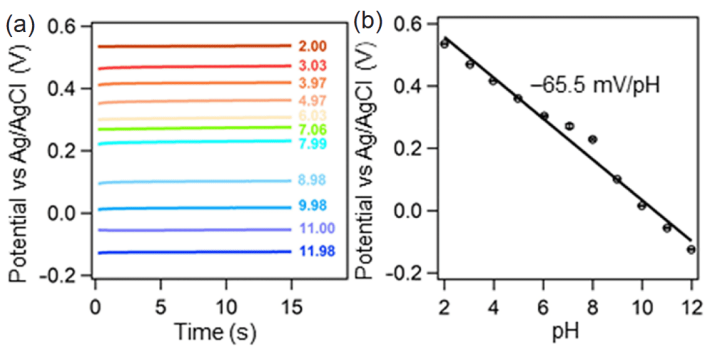

图 2 氧化铱纳米颗粒修饰碳纤维电极在不同 $\mathrm{pH}$ 溶液中的 开路电位检测结果(a)和对应线性拟合结果(b). 支持电解质 为 $0.1 \mathrm{~mol} / \mathrm{L} \mathrm{KCl}$ (网络版彩图)

Figure 2 The open circuit potential (OCP) results of the iridium oxide nanoparticles modified carbon fiber microelectrode at different $\mathrm{pH}$ (a) and the linear fitting result (b). The supporting electrolyte is $0.1 \mathrm{~mol} / \mathrm{L}$ $\mathrm{KCl}$ (color online).

$E=E_{\mathrm{a}}^{\Theta}+\frac{2.303 R T}{n F} \lg \left(a_{\mathrm{H}^{+}}\right)^{x}=E_{\mathrm{a}}^{\Theta}-\frac{2.303 x R T}{n F} \mathrm{pH}$

其中 $E_{\mathrm{a}}^{\Theta}$ 为标准电势, $a_{\mathrm{H}^{+}}$为氢离子活度, $R$ 为摩尔气体 常数 $(8.314 \mathrm{~J} /(\mathrm{mol} \mathrm{K})), T$ 为温度 $(\mathrm{K}), n$ 为反应的电子转 移数, $F$ 为法拉第常数 $(96485 \mathrm{C} / \mathrm{mol})$. 氧化铱氢氧化物 在溶液中的氧化还原反应方程如公式(2)所示:

$2\left[\mathrm{IrO}_{2}(\mathrm{OH})_{y}\right]^{y-}(\mathrm{s})+x \mathrm{H}^{+}(\mathrm{w})+2 \mathrm{e}^{-}$
$=\left[\mathrm{Ir}_{2} \mathrm{O}_{3}(\mathrm{OH})_{3}\right]^{3-}(\mathrm{s})+(2 y-2) \mathrm{H}_{2} \mathrm{O}(\mathrm{w})$

以氧化铱为功能材料的 $\mathrm{pH}$ 电化学传感器对 $\mathrm{pH}$ 的 响应主要来自 $\operatorname{Ir}(\mathrm{IV})$ 和 $\mathrm{Ir}(\mathrm{III})$ 间的价态变化, 因此电子 转移数 $n=2$, 结合实验测得的氧化铱纳米颗粒修饰电 极对 $\mathrm{pH}$ 响应的灵敏度为 $65.5 \mathrm{mV} / \mathrm{pH}$, 计算得到参与反 应的氢离子数 $x=2.2$. 电化学方法修饰在碳纤维电极表 面的氧化铱 $\left(\mathrm{IrO}_{2}\right)$ 水化氢氧化物结合的氢氧根数量 $y$ 与 制备条件相关，根据反应方程的电荷平衡，计算得到 $y=1.6$. 因此氧化铱修饰碳纤维电极在溶液中的反应方 程式为:

$2\left[\mathrm{IrO}_{2}(\mathrm{OH})_{1.6}\right]^{1.6-}(\mathrm{s})+2.2 \mathrm{H}^{+}(\mathrm{w})+2 \mathrm{e}^{-}$
$=\left[\mathrm{Ir}_{2} \mathrm{O}_{3}(\mathrm{OH})_{3}\right]^{3-}(\mathrm{s})+1.2 \mathrm{H}_{2} \mathrm{O}(\mathrm{w})$

最后，我们通过循环检测实验与连续检测实验评 估传感器的稳定性. 其中, 循环检测实验将电极反复 浸泡于 $\mathrm{pH}=3$ 和 $\mathrm{pH}=10$ 的 $0.1 \mathrm{~mol} / \mathrm{L} \mathrm{KCl}$ 溶液中进行开路 电位检测. 实验结果如图3所示, 在 10 轮循环检测过程 中电极对 $\mathrm{pH}=3$ 和 $\mathrm{pH}=10$ 的溶液的响应平均偏差分别 为7.39和 $6.93 \mathrm{mV}(n=10)$. 连续检测实验结果如图 $\mathrm{S} 3 \mathrm{~b}$ 


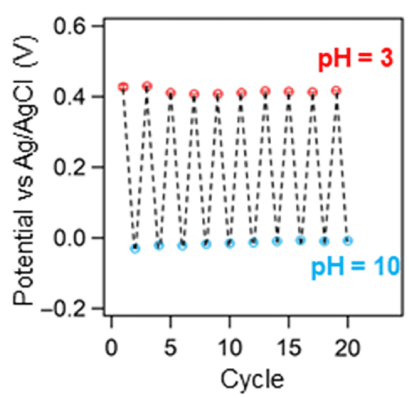

图 3 氧化铱纳米颗粒修饰碳纤维电极的 $\mathrm{pH}$ 循环检测结果 (网络版彩图)

Figure 3 The cycle open circuit potential detection results of iridium oxide nanoparticles modified carbon fiber microelectrode in $0.1 \mathrm{~mol} / \mathrm{L}$ $\mathrm{KCl}$ solution $(\mathrm{pH}=3$ and $\mathrm{pH}=10)$ (color online).

所示, 在 $\mathrm{pH}=3$ 和 $\mathrm{pH}=10$ 的 $0.1 \mathrm{~mol} / \mathrm{L} \mathrm{KCl}$ 溶液中, 电极对 $\mathrm{pH}$ 的响应在 $600 \mathrm{~s}$ 内基本不变. 因此我们认为氧化铱纳 米颗粒修饰碳纤维电极在较短时间的测试中具有较好 的稳定性.

\section{3 植物组织的 $\mathrm{pH}$ 检测}

为了进一步探究氧化铱纳米颗粒修饰碳纤维电极 在实际应用中的潜力，我们以橘子和西瓜为实际样品， 对两种水果组织内的 $\mathrm{pH}$ 进行了检测. 实验装置示意图 如图4所示, 我们用立体定位仪将氧化铱纳米颗粒修饰 碳纤维电极和银/氯化银微参比电极同时植入水果组 织中，电极间距约为 $5 \mathrm{~mm}$. 氧化铱纳米颗粒修饰碳纤 维电极的开路电压用电化学工作站记录(实验装置实 物图与开路电位检测结果见图S4). 结果如表1所示, 使 用氧化铱纳米颗粒修饰碳纤维电极测得西瓜和橘子果 肉组织内 $\mathrm{pH}$ 值分别为 $5.60 \pm 0.13$ 和 $3.40 \pm 0.27$. 该结果与 用玻璃电极测得的西瓜汁和橘子汁 $\mathrm{pH}$ 值吻合, 实验说

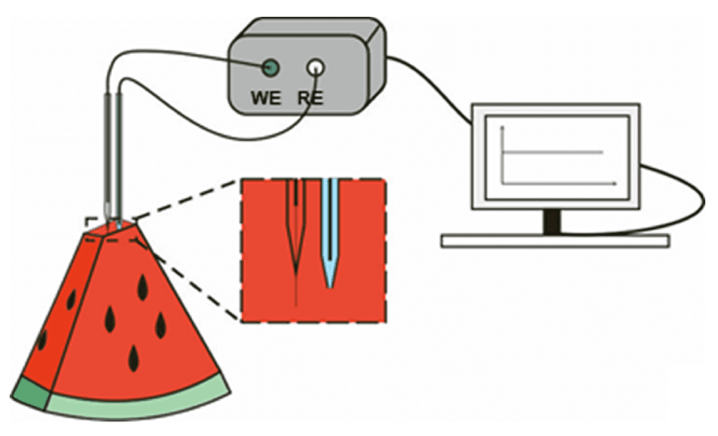

图 4 植物组织 $\mathrm{pH}$ 在线检测装置图 (网络版彩图)

Figure 4 The scheme of the device for the $\mathrm{pH}$ detection in the plant tissue (color online).

表 1 植物组织 $\mathrm{pH}$ 检测结果

Table 1 The $\mathrm{pH}$ detection results of IONPs/CFME in plant tissue

\begin{tabular}{ccc}
\hline 样品 & 实际 $\mathrm{pH}$ 值 $^{\mathrm{a})}$ & 实验 $\mathrm{pH}$ 值 \\
\hline 橘子 & $3.37 \pm 0.03$ & $3.40 \pm 0.27$ \\
西瓜 & $5.63 \pm 0.01$ & $5.60 \pm 0.13$ \\
\hline
\end{tabular}

a) 实际 $\mathrm{pH}$ 值通过商用 $\mathrm{pH}$ 计测得 $(n=3)$.

明我们发展的氧化铱纳米颗粒修饰碳纤维电极具备应 用前景.

\section{4 结论}

本文通过在碳纤维微电极表面电化学修饰氧化铱 纳米颗粒的方法构建了一种微型pH传感器，该传感器 具有灵敏度高、检测范围宽、稳定性高等优点. 为进 一步研究氧化铱纳米颗粒修饰碳纤维电极的应用潜 力, 我们以水果为实际样品, 对水果组织的 $\mathrm{pH}$ 进行了 检测, 测得的结果与实际结果基本一致.

致谢＼cjkstart浙江大学化学系化学仪器中心陈芳女士协助扫描电子显微镜测试, 在此表示感谢.

\section{补充材料}

本文的补充材料见网络版chemen.scichina.com. 补充材料为作者提供的原始数据, 作者对其学术质量和内容负责.

\section{参考文献}

1 Buck RP, Rondinini S, Covington AK, Baucke FGK, Brett CMA, Camoes MF, Milton MJT, Mussini T, Naumann R, Pratt KW, Spitzer P, Wilson GS. Pure Appl Chem, 2002, 74: 2169-2200

2 Zhang H, Wang J, Ye S. Anal Chim Acta, 2008, 606: 112-118

3 Kress-Rogers E. Trends Food Sci Tech, 1991, 2: 320-324 
4 Lin HJ, Szmacinski H, Lakowicz JR. Anal Biochem, 1999, 269: 162-167

5 Banna MH, Najjaran H, Sadiq R, Imran SA, Rodriguez MJ, Hoorfar M. Sens Actuat B-Chem, 2014, 193: 434-441

6 Qin Y, Kwon HJ, Howlader MMR, Deen MJ. RSC Adv, 2015, 5: 69086-69109

7 Li Z, Wu S, Han J, Han S. Analyst, 2011, 136: 3698-3706

8 Nakata S, Arie T, Akita S, Takei K. ACS Sens, 2017, 2: 443-448

9 Manjakkal L, Szwagierczak D, Dahiya R. Prog Mater Sci, 2020, 109: 100635

10 MacInnes DA, Dole M. J Am Chem Soc, 1930, 52: 29-36

11 Rondinini S. Anal BioAnal Chem, 2002, 374: 813-816

12 Manjakkal L, Cvejin K, Kulawik J, Zaraska K, Szwagierczak D, Socha RP. Sens Actuat B-Chem, 2014, 204: 57-67

13 Manjakkal L, Cvejin K, Bajac B, Kulawik J, Zaraska K, Szwagierczak D. Electroanalysis, 2015, 27: 770-781

14 Chen PY, Yin LT, Shi MD, Lee YC. Life Sci J, 2013, 10: 3132-3136

15 Vieira NCS, Figueiredo A, Faceto AD, de Queiroz AAA, Zucolotto V, Guimarães FEG. Sens Actuat B-Chem, 2012, 169: 397-400

16 Zhou L, Cheng C, Li X, Ding J, Liu Q, Su B. Anal Chem, 2020, 92: 3844-3851

17 da Silva GM, Lemos SG, Pocrifka LA, Marreto PD, Rosario AV, Pereira EC. Anal Chim Acta, 2008, 616: 36-41

18 Cao H, Landge V, Tata U, Seo YS, Rao S, Tang SJ, Tibbals HF, Spechler S, Chiao JC. IEEE Trans Biomed Eng, 2012, 59: 3131-3139

19 Zea M, Moya A, Fritsch M, Ramon E, Villa R, Gabriel G. ACS Appl Mater Interfaces, 2019, 11: 15160-15169

20 Li S, Xu Y, Chen Y, Li W, Lin L, Li M, Deng Y, Wang X, Ge B, Yang C, Yao S, Xie J, Li Y, Liu X, Ma D. Angew Chem Int Ed, 2017, 56: $10761-10765$

21 Marzouk SAM, Ufer S, Buck RP, Johnson TA, Dunlap LA, Cascio WE. Anal Chem, 1998, 70: 5054-5061

22 Yamanaka K. Jpn J Appl Phys, 1989, 28: 632-637

23 Gao J, Xu CQ, Hung SF, Liu W, Cai W, Zeng Z, Jia C, Chen HM, Xiao H, Li J, Huang Y, Liu B. J Am Chem Soc, 2019, 141: 3014-3023

Burke LD, Mulcahy JK, Whelan DP. J Electroanal Chem Interfacial Electrochem, 1984, 163: 117-128

\title{
Iridium oxide nanoparticles modified carbon fiber microelectrodes for pH detection
}

\author{
Xinru Li, Lin Zhou, Bin Su ${ }^{*}$ \\ Department of Chemistry, Zhejiang University, Hangzhou 310058, China \\ *Corresponding author (email: subin@zju.edu.cn)
}

\begin{abstract}
H}$ detection plays an important role in agriculture, food processing, environment protection and disease diagnosis. Electrochemical methods are widely used in $\mathrm{pH}$ sensing owing to their fast response, high sensitivity and simple to operate. But commercial $\mathrm{pH}$ sensors can only work in relatively stable in vitro environment rather than in vivo environment because of their large size and fragile proton sensitive membrane. In this work, we modify iridium oxide nanoparticles on the carbon fiber microelectrode, and fabricate a miniature, implantable solid $\mathrm{pH}$ sensor. The $\mathrm{pH}$ sensor has super-Nernstian $\mathrm{pH}$ response (its sensitivity can reach $65.5 \mathrm{mV} / \mathrm{pH}$ ), wide linear range $(\mathrm{pH} \approx 2-12)$ and high stability. Finally, we implant the $\mathrm{pH}$ sensor into the fruit tissue and achieve the detection of $\mathrm{pH}$ in the fruit.
\end{abstract}

Keywords: Iridium oxide, implantable, $\mathrm{pH}$, super-Nernstian, fruit

doi: $10.1360 /$ SSC-2020-0197 